"The British Gynæcological Journal."-The May and August parts (xxi and xxii, 1890), contain the usual variety of interesting matter. Cancer of the uterus and its treatment occupy a large portion of the May part. Dr. Macan strongly advocated vaginal extirpation in preference to less radical measures. Dr. Jessett thought that in some cases high amputation of the cervix is sufficient. Dr. Bantock thought that if he saw a case early enough be might perform amputation of the cervix, but in the condition usually met with he would advocate extirpation. Dr. Inglis Parsons advised the trial of a strong electric current-five to six hundred milliampères for, say, thirty seconds - on the ground that it killed the cells, and brought on atrophy, degeneration, and subsequent absorption. The difficulty acknowledged by all was that of recognition early enough in the disease, many of them (Bantock, Reeves, \&c.) refusing to recognise the microscope as of any aid in the diagnosis.

Another important discussion was on pelvic abscess. R. T. Smith reviewed the various kinds of this abscess, some originating in cellulitis post partum, or after operation, or from chill during menstruation, others in peritonitis from affections of tube or ovary; the former tending along the lines of connective tissue towards the surface, while the latter, forming encysted tumours, tend to perforate adjacent organs.

Mayo Robson, in an important paper, discussed the modes of treatment by (1) aspiration-efficient in small and localised abscesses; (2) subperitoneal laparotomy -only safe when the abscess is approaching the side of the pelvis ; and (3) abdominal section - the method of widest application. Mr. Robson narrated examples of all these methods.

Dr. Bantock asserted that he had only seen one pelvic abscess arising from cellulitis, all the others having arisen from mischief affecting the tube or tube and ovary.-J. K. K.

\title{
DISEASES OF THE EYE.
}

\section{By FREELAND FERGUS, M.B.}

Transactions of the Ophthalmological Society. -. The tenth volume of the Transactions of the $\Theta$ phthalmological Society has just beentissued, and in our present number we propose giving a short synopsis of its contents.

The President's address, given by Dr. Hughlings Jackson, is an appeal to physicians and to ophthalmologists to work hand in hand in the investigation of diseases of the nervous system. He says, "Unless the physician uses the ophthalmoscope by routine, he will often enough overlook the best evidence-and, I am convinced, in some cases the only decisive evidence-of gross organic disease of the brain there is to be had; and if, as is often the case in a physician's practice, sight be good, he will not surmise that there is anything wrong with his patient's optic nerves." Again, "I urge young physicans to study eye diseases at an ophthalmic hospital, or at an ophthalmic department of a general hospital."

The following remarks also are pregnant with meaning:- "It is very remarkable that many patients with optic neuritis die suddenly or rapidly, and when seemingly in fair general health, sometimes at work, certainly most unexpectedly; I have often urged this on the attention of physicians. In some cases of cerebral tumour the patient may be acutely ill; he may have an illness very like tubercular meningitis in many of its symptoms; there may be slow and unrhythmical pulse and irregular respiration, there are retracted abdomen, constipation and vomiting. I suppose that when a patient who has post neuritic atrophy tells us that his sight failed after bilious or gastric fever, he has had such an acute illness." Jackson indicates a belief, that when a tumour causes optic neuritis it is trying to make the patient blind, that when it produces such "vital" symptoms it is trying to kill him. He quotes Buzzard as indicating that these vital symptoms depend upon morbid changes 
in nerves, such as the pneumogastric, perfectly comparable with the changes seen with the ophthalmoscope in optic neuritis.

The "Pathogeny of Concomitant Squint" was the subject of the Bowman lecture by Hansen Grut, of Copenhagen. It is certainly one of the clearest and best contributions which has appeared for many a long day on this important question. The lecture is of considerable length, and the argument seems irresistible, and those who are interested in the matter ought to read it for themselves; we can only mention very briefly a few of the more important points.

He starts with the statement that the concomitancy of lateral and convergent movements is a result of habit and exercise-i.e., that there are no special centres for such movements, but that these movements are the result of acquired habit. Again, squint, according to the author, must depend either on some defective anatomical position or on some abnormality of the innervation. In his opinion, the vast majority of cases are due to the second cause. Having stated these preliminaries, the next thing considered by Grut is the anatomical position of rest of the eyes. This he believes to be one of divergence, and the five reasons which he assigns for this belief seem to us very conclusive. This opinion, of course, is the very opposite from Stilling's ; but we think that Grut has quite succeeded in showing that Stilling's views are not correct. The patient does not, as a rule, assume anatomical position of rest, for then there would be diplopia, but a functional one of parallelism. Grut then recalls the experiments and riews of the immortal Donders as to the meter angle and the accommodation moving to equal amounts in emmetropia, and makes the three following very suggestive statements :-

"In emmetropia, nearly always perfect harmony; the meter angle and accommodation correspond. The exceptions : convergence, being in excess of accommodation, is exceedingly rare."

"In hypermetropia, harmony between meter angle and accommodation, which would produce latent convergent squint, is certainly not the rule, but infinitely more frequent than in emmetropia."

In the main, Grut's views are those of Donders, but with considerable modification. "When the state of refraction and accommodation induce a tendency to squint, it is a trick, an art, to resist squinting, and it has to be learned."

This trick certain persons never learn, and hence they squint. "Convergent strabismus originates, and continues as the result of, an innervation which effects in the interni a shortening exceeding in amount that which is desirable." "Divergent strabismus is the expression of a relaxation of convergence innervation, which permits of the eye taking up the anatomical position of rest."

Habit will make the convergent squint of hypermetropia permanent-i.e., when the patient is not able to accommodate without converging; and there are very many in this condemnation.

In speaking of divergent squint in myopia, Grut at once throws over the idea of its being preponderance of the external recti over the internal. He believes it to be the causation of convergence, allowing the eyes to take their position of anatomical rest.

These are the chief points of a most interesting and lucid paper. The rest of it is occupied with a criticism of the views of Schmeigger, Alf. Græfe, Stilling, and others.

Dr. Rockliffe records an interesting case of cephalic tetanus. This somewhat rare malady came on after a slight trivial wound of the left orbit. There were ptosis and facial paralysis of the same side of the face, with trismus and slight pharyngeal and respiratory spasms.

The treatment consisted in opening up and cleaning the wound under chloroform, and in giving frequently repeated doses of bromide of potassium and chloral. The patient ultimately recovered.

"Lymphoma of the Conjunctiva" is the subject of a very suggestive paper by Dr. Thomas Reid, in which the author attempts to regard various morbid conditions of the conjunctiva as modifications of normal structure. He says- 
"An examination of the specimens shows that the presence of lymphoid tissue; or perbaps more correctly lymphoid infiltration, is a frequent if not constant pathological condition in certain forms of conjunctivitis."

The follicle, Dr. Reid believes, is formed secondarily to the lymphoid infiltration. He describes the changes which occur in the follicle, leading either to the discharge of its contents or to their absorption. These follicles are found both in follicular conjunctivitis and in trachoma. "If it be admitted that in all cases the presence of lymphoid tissue is at one stage or other a constant element, the distinction that has been drawn between follicular and trachomatous conjunctivitis is more a clinical than a pathological one." Again, "Whatever hastens this eliminative process will promote the natural cure of granular ophthalmia, and in our hands stimulants and absorbents have always proved distinctly more serviceable than any form of astringent."

Priestley Smith writes an article in which he shows, from carefully collected statistics, that glaucoma is very frequently associated with a diminution in the size of the cornea. From his figures it would also appear that the size of the cornea does not vary much with the state of the refraction.

"Tuberculosis of the Iris."-Dr. Hill Griffith describes a case of this somewhat rare affection. The tumour grew near the periphery of the iris, involving the ciliary body. The eye was enucleated, and the child died nine months afterwards from tubercular meningitis.

"Glaucoma after Cataract Extraction," is the subject of a paper by Mr. E. Teacher Collins. He describes the results of the examination of ten eyes, and finds that in nine of them there was adherence of the lens capsule to the corneal wound; in the remaining case the iris was attached. Such a condition of matters must lead to a certain plugging of the iris angle. This of itself seems sufficient in many cases to cause glaucoma. In others, he thinks that inflammatory conditions-e.g., kerako-iritis, or the irritation of the ciliary processes, caused by dragging of the adherent capsule, are also important factors. The practical point seems to be to get the wound to heal as quickly as possible, and to see that it is free both from iris and capsule.

"Optic Nerve Atrophy in Smokers."-Mr. Lawford describes several cases in which he felt justified in giving a favourable prognosis when he believed that he was dealing with tobacco amblyopia. In the cases under discussion, however, the favourable view he was inclined to take was by no means justified by the result, for the nerves steadily atrophied and the sight did not materially improve. He is inclined to think that the tobacco, at least, has something to do with the condition of matters. The only point in which these cases differed from ordinary ones is, that not only did they present the usual central scotoma, but also a retraction of the field of vision at the periphery.

A discussion on the artificial ripening of immature cataracts, and two cases of hemianopsia, complete the more important contents of the volume.

\section{Books, Pamphlets, Éc., Received.}

On Severe Vomiting during Pregnancy, by Graily Hewitt, M.D. London : Longmans, Green \& Co. 1890.

Lectures on Diabetes, by Robert Saundby, M.D. With Illustrations. Bristol : John Wright \& Co. 1891.

The Medical Annual and Practitioner's Index. 1891. Ninth year. Bristol : John Wright \& Co.

Transactions of the Royal Academy of Medicine 'in Ireland. Vol. VIII. Edited by Wm. Thomson, M.A., F.R.C.S. Dublin: Fannin \& Co. 1890. 